\title{
Differences in radiotherapy application according to regional disease characteristics of hepatocellular carcinoma
}

\author{
Chai Hong Rim ${ }^{1,2}$ \\ Department of Radiation Oncology, ${ }^{1}$ Korea University Ansan Hospital, Ansan; ${ }^{2}$ Korea University Medical College, Seoul, Korea
}

Received Mar. 25, 2021

Revised May 25, 2021

Accepted Jun. 1, 2021
There are differences in opinion regarding the application of external beam radiotherapy in the treatment of hepatocellular carcinoma. Some major guidelines state that external beam radiotherapy is yet to attain a sufficient level of evidence. However, caution should be exercised when attempting to understand the clinical need for external beam radiotherapy solely based on the level of evidence. Previously, external beam radiotherapy had low applicability in the treatment of hepatocellular carcinoma before computed tomography-based planning was popularized. Modern external beam radiotherapy can selectively target tumor cells while sparing normal liver tissues. Recent technologies such as stereotactic body radiotherapy have enabled more precise treatment. The characteristics of hepatocellular carcinoma differ significantly according to the regional etiology. The main cause of hepatocellular carcinoma is the hepatitis B virus. It is commonly diagnosed as a locally advanced tumor but with relatively preserved hepatic function. The majority of these hepatocellular carcinoma cases are found in the East Asian population. Hepatocellular carcinoma caused by hepatitis $C$ virus or other benign hepatitis tends to be diagnosed as a less locally aggressive tumor but with deteriorated liver function. The western world and Japan tend to have patients with such causes. External beam radiotherapy has been more commonly performed for the former, although the use of external beam radiotherapy in the latter might have more concerns with regard to hepatic toxicity. This review discusses the above subjects along with perspectives regarding external beam radiotherapy in recent guidelines. (J Liver Cancer 2021;21:113-123)

Keywords: Hepatocellular carcinoma; External beam radiation therapy; Radiotherapy; Liver neoplasm; Guidelines

\section{INTRODUCTION}

Liver cancer is the second leading cause of cancer-related deaths worldwide. Hepatocellular carcinoma (HCC) accounts for approximately $85 \%$ of all liver cancers, compris-

\section{Corresponding author: Chai Hong Rim}

Department of Radiation Oncology, Korea University Ansan Hospital, 123 Jeokgeum-ro, Danwon-gu, Ansan 15355, Korea

Tel. +82-31-412-6850, Fax. +82-31-412-6851

E-mail: crusion3@naver.com ing the absolute majority of such cancers. ${ }^{1}$ The causes of HCC include viral infection, chronic alcohol consumption, and fatty liver disease, the proportions of which differ by region. The vast majority of liver cancers occur in East Asia. Most HCCs in this region are mainly caused by chronic hepatic inflammation due to hepatitis B virus infection. In contrast, the main causes of HCC in Western countries are hepatitis $\mathrm{C}$ virus infection, alcoholic hepatitis, and liver cirrhosis related to metabolic syndrome. These etiologies affect not only the incidence but also the nature of the disease as well as the treatment prognosis. ${ }^{2}$ 
The surgical approach has been the conventional method for the radical treatment of HCC. For unresectable HCC, various local treatment options have been used. For early and small HCCs, radiofrequency ablation (RFA) is used as a noninvasive surrogate for surgical resection and has demonstrated oncologic outcomes comparable to those of surgery. ${ }^{3,4}$ Transarterial chemoembolization (TACE) is the most commonly used local treatment for HCC and has a wide range of palliative indications, from early cases to intermediate cases. ${ }^{5,6}$

External beam radiation therapy (EBRT) was previously contraindicated for patients with HCC owing to vessel-occlusive toxicity (referred to as radiation-induced liver disease [RILD]) after whole-liver irradiation. ${ }^{7}$ However, as it is now possible to selectively irradiate tumors while reducing the exposure of normal liver tissues using computed tomography (CT)-based planning, pioneering researchers have begun using EBRT in the treatment of HCC. ${ }^{8}$ With the development of image guidance and conformal radiotherapy technologies, stereotactic body radiotherapy (SBRT), which delivers a high dose of radiation in smaller $(<5-10)$ fractions, is being used to treat small HCCs as a form of ablative treatment. ${ }^{9,10}$

However, while TACE and RFA have relatively common indications worldwide based on the results of randomized studies, EBRT was prescribed based on a case series reflecting clinical experiences in practice. Geographically, EBRT is commonly used in East Asian countries, where locally advanced and vessel invasive cases are common. However, it is not commonly used in Western countries. ${ }^{2}$ In addition, the indications for EBRT in the clinical guidelines issued in various countries and by different associations are not uniform; they vary significantly. ${ }^{11,12}$ This review discusses the disease characteristics of HCC by region and indications for EBRT based on clinical practice and various treatment guidelines.

\section{APPLICATION AND FEASIBILITY OF EBRT FOR HCC}

Before CT-based radiation planning was widely adopted in the 1980 and 1990s, EBRT based on plain X-ray films (the so-called two-dimensional EBRT) was performed in clinics. Anatomical bony landmarks were commonly used to delin- eate treatment fields. Therefore, these fields were usually wider than they are today, causing more complications. Concerning HCC, it was difficult to differentiate the tumorous region from the normal liver tissues; hence, treatment in those days involved the irradiation of nearly the entire liver (Fig. 1). ${ }^{13}$

When the radiation dose to the entire liver exceeds $30 \mathrm{~Gy}$, the rate of RILD increases rapidly. RILD is a complication typically involving anicteric hepatomegaly, ascites, and elevation of alkaline phosphatase levels and manifests following the obliteration of the central vein of the hepatic lobule, retrograde congestion, and consequent hepatic necrosis. ${ }^{14}$ Classically, according to Ingold et al., ${ }^{15}$ one of eight patients (17\%) developed RILD after receiving 30-35 Gy of wholeliver irradiation, whereas 12 of 27 (44\%) patients experienced RILD after receiving >35 Gy. The RTOG 84-05 dose-escalation study revealed that none of the 122 patients experienced RILD after irradiation with 27-30 Gy of EBRT. Whereas, five of $51(9.8 \%)$ patients developed RILD after receiving $33 \mathrm{~Gy}{ }^{16}$ Emami et al. ${ }^{17}$ estimated that the tolerance dose (calculated as a $5 \%$ risk of RILD at 5 years post-treatment, or the "TD $\left.5 / 5^{\prime \prime}\right)$ to the whole liver was $30 \mathrm{~Gy}$. Since doses to the entire liver of $<30$ Gy were insufficient to obtain a significant tumor response, EBRT was commonly contraindicated in the treatment of HCC. ${ }^{18}$

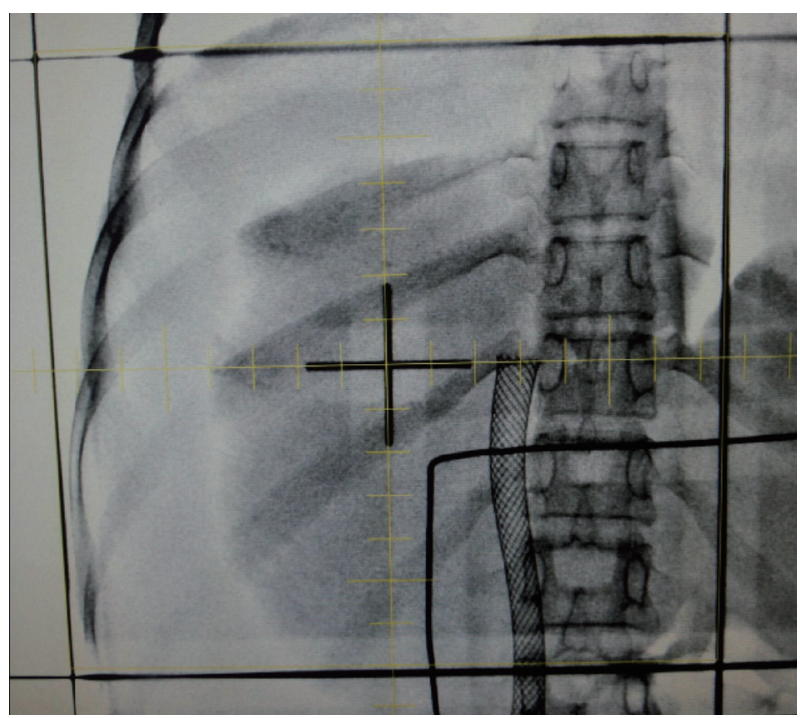

Figure 1. Two-dimensional radiotherapy portal image of almost the entire liver. Adopted from J Egypt Natl Canc Inst 2017;29:105-108. ${ }^{13}$ 
However, the development of an EBRT technique based on CT-based computerized planning enabled partial irradiation of the tumor while reducing bystander irradiation to the normal liver. EBRT based on CT planning is referred to as three-dimensional conformal radiotherapy (3DCRT) to distinguish it from two-dimensional radiotherapy. Additional advanced modalities, including SBRT and intensity-modulated radiotherapy, are also based on CT-based planning. Furthermore, computerized planning enabled the quantitative assessment of the effect of radiation dose on each organ and target using a dose-volume histogram (Fig. 2). Researchers have attempted to identify dosimetric factors that can be

(A)

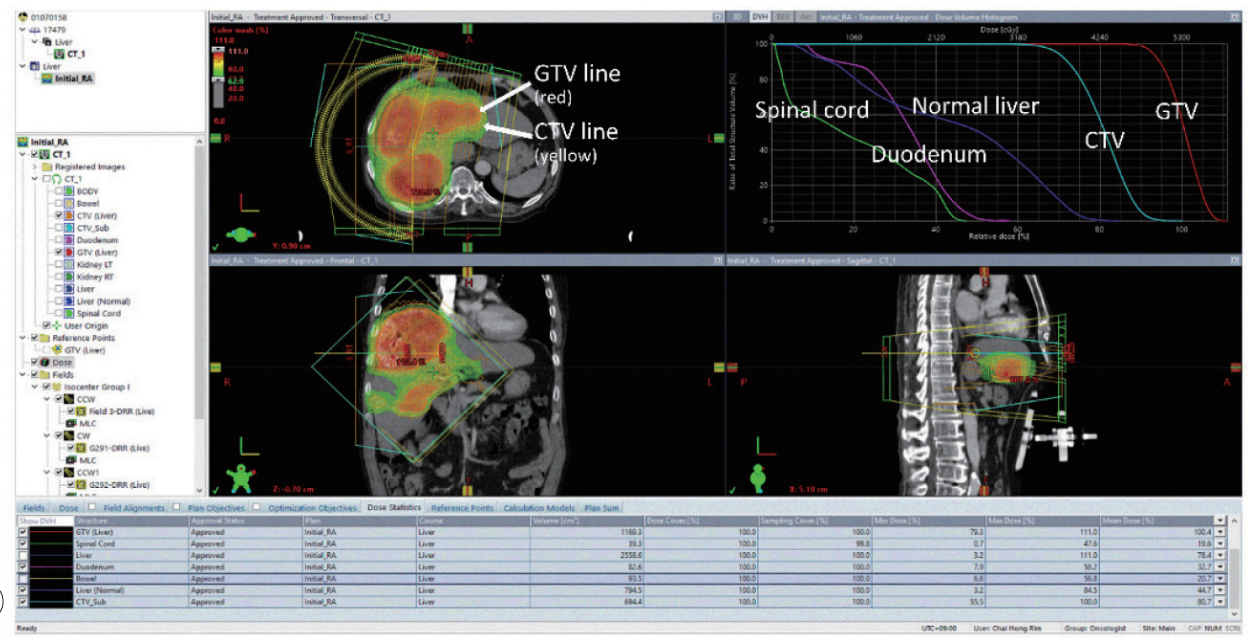

(B)

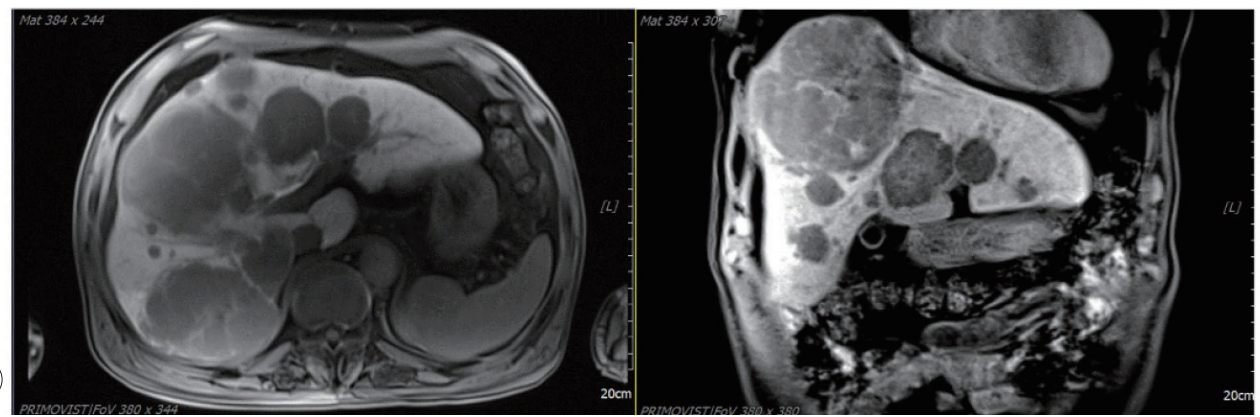

(C)

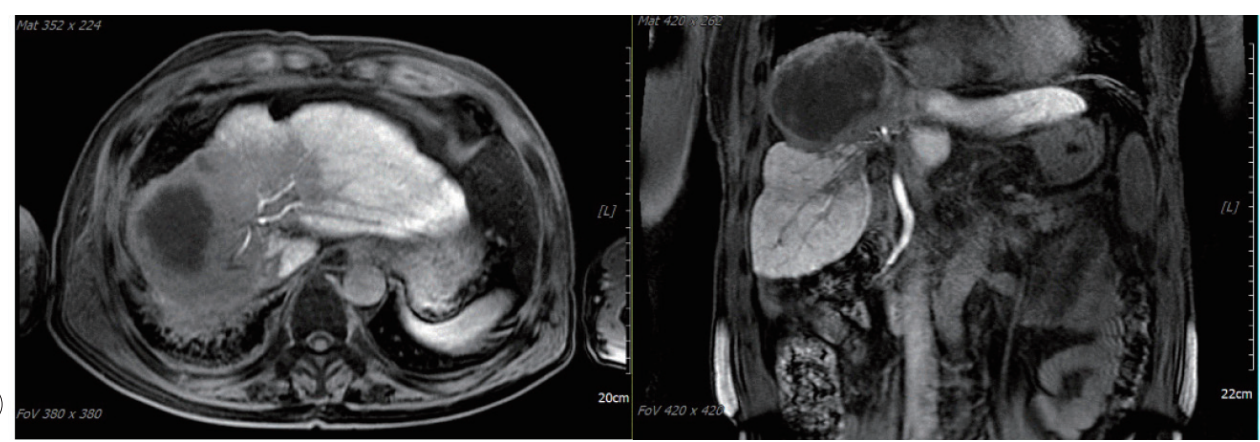

Figure 2. Computerized tomography-based radiotherapy planning and a case of locally advanced hepatocellular carcinoma (HCC) with portal vein thrombosis (PVT). (A) A color wash of distribution of radiation dose (upper left, lower left, and lower right) and dose-volume histogram (upper right). We planned to save at least 70\% of normal liver (left lobe) to be irradiated under $30 \mathrm{~Gy}$. (B) Pre-radiotherapy images: multiple HCCs dominantly located in the right lobe involving right PVT. Child Pugh-score was A6. (C) One year after $53 \mathrm{~Gy} / 20 \mathrm{~F}$ external radiotherapy and 3 times of transarterial chemoembolization. The tumors were controlled without evidence of viable tumors, with a necrotic change of the main tumor and hypertrophy of the left lobe. The Child-Pugh score was A6. The patient was further followed up for another year and died due to gastrointestinal bleeding. GTV, gross tumor volume; CTV, clinical target volume. 
used to optimize the clinical application of radiotherapy. Among them, the mean dose to the liver and liver volume irradiated with $>30 \mathrm{~Gy}\left(\mathrm{~V} 30_{\mathrm{Gy}}\right)$ are the most reliable factors to date.

Dawson et al. ${ }^{19}$ from the University of Michigan reported that the mean liver dose of patients who did not develop RILD ( $\mathrm{n}=184$ ) was 31.3 Gy (range: 14.9-44.0 Gy), whereas that of patients who developed RILD ( $\mathrm{n}=19$ ) was $37.0 \mathrm{~Gy}$ (range: 31.6-43.7 Gy). They estimated a dose-response relationship in which the risk of RILD increased by approximately $4 \%$ for every gray above the mean dose threshold of $30 \mathrm{~Gy}$ and recommended a mean liver dose of $<28 \mathrm{~Gy}$ in 2 Gy per fraction in their subsequent review. ${ }^{20}$ Kim et al. ${ }^{21}$ reported that the $\mathrm{V} 30_{\mathrm{Gy}}$ was the most significant dosimetric factor for predicting radiation-induced hepatic toxicity. When the $\mathrm{V} 30_{\text {Gy }}$ exceeded $60 \%$ of the whole liver, the risk of serious hepatic toxicity steeply increased (grade 2 or worse toxicity: $2.4 \%$ vs. $55 \%$ with a threshold $\mathrm{V} 30_{\mathrm{Gy}}$ of $60 \%$ ). Therefore, a $\mathrm{V} 30_{\mathrm{Gy}}$ of $<60 \%$ of the liver volume was recommended to avoid serious hepatic toxicity. Regarding SBRT, the guidance that at least $700 \mathrm{~mL}$ of the liver volume should be irradiated below $15 \mathrm{~Gy}$ is commonly used. A volume of $700 \mathrm{~mL}$ was suggested to be sufficient after partial hepatectomy, and a dose of $15 \mathrm{~Gy}$ (which is biologically equivalent to $24 \mathrm{~Gy}$ in conventional 2 Gy per fraction) was deemed safe. .2,23 $^{2}$

Practical guidance using quantitative indicators such as the $\mathrm{V} 30_{\mathrm{Gy}}$ and mean liver dose is also recommended in treatment guidelines from Asian countries. ${ }^{2,24,25}$ In three guidelines, including those from a nationally representative liver cancer study group from China, ${ }^{25}$ Hong Kong, ${ }^{2}$ and South Korea, ${ }^{24}$ it was recommended that at least $700 \mathrm{~mL}$ of the liver volume should be unirradiated or irradiated with a limited dose $(<15 \mathrm{~Gy})$ when applying SBRT. In the guidelines from South Korea, it is also stated that $>40 \%$ of the liver volume should be irradiated at $<30 \mathrm{~Gy} .{ }^{24}$ Many researchers also refer to the liver tolerance dose recommended by the Quantitative Analysis of Normal Tissue Effects in the Clinic review, which proposes a TD 5/5 of $<30-32$ Gy for RILD. ${ }^{26}$

The application of 3DCRT, based on CT-based planning, also reduced the risk of gastrointestinal toxicity associated with EBRT. The radiation tolerance dose of the small intes- tine is low, with a TD 5/5 of 40-50 Gy. ${ }^{17}$ In a previous twodimensional treatment, where only the bony landmark and luminal air shadow were referenced, it was not known how much radiation the intestine was exposed to. The alimentary tract comprises a series of organs; thus, even minor damage can greatly impair organ function and cause systemic damage. However, modern EBRT can quantitatively measure the amount of radiation received by the small intestine or duodenum (Fig. 2). It has been reported that the treatment response rate of HCC also improves as the dose of radiation therapy is increased. ${ }^{27,28}$ Therefore, a dose-escalation approach using CT-based planning can increase tumor controllability and reduce unexpected toxicity. In particular, the use of SBRT, which is used for high-dose short-term treatment, enables curative treatment for early HCCs. ${ }^{10}$

In many studies conducted after 3DCRT, radiotherapy for liver cancer has been relatively performed safely. According to a meta-analysis by our team, of 2,513 patients with HCC who underwent EBRT targeting portal vein thrombosis and/ or intrahepatic tumors, grade $\geq 3$ gastrointestinal or hepatic complications were rarely reported; they occurred in $<10 \%$ of patients in most of the included studies. ${ }^{29}$ In another meta-analysis performed by our colleagues, of a clinical series involving 1,950 patients who underwent SBRT for HCC, the pooled rates of grade $\geq 3$ gastrointestinal and hepatic complications were $3.9 \%$ and $4.7 \%$, respectively. ${ }^{10}$ These favorable safety profiles may have been a result of the aforementioned quantitative EBRT dose guidance, which was enabled by CTbased planning.

In summary, EBRT based on CT-based planning has become a feasible modality in the treatment of HCC despite previous contraindications, as it enables selective irradiation of tumors. Quantitative dosimetric indices (e.g., the mean liver dose or ${\mathrm{V} 30_{\mathrm{Gy}}}_{\mathrm{y}}$ assessed by computerized planning have further enabled objective clinical guidance for the safe application of EBRT.

\section{VARYING CHARACTERISTICS OF HCC AND APPLICATION OF EBRT}

The major etiologies of HCC include hepatitis B or C virus 
infection, alcoholic hepatitis, and fatty liver associated with metabolic syndrome. Among these factors, hepatitis B virus infection accounts for the largest proportion of cases, as it is the cause of approximately half of HCC cases worldwide. ${ }^{30}$ HCC caused by hepatitis B virus infection is particularly prevalent in East Asian countries. Moreover, hepatitis $\mathrm{C}$ virus infection is the main cause of HCC in Japan, while alcoholic liver disease, non-alcoholic fatty liver disease, and hepatitis $\mathrm{C}$ virus are major causes of HCC in Western countries, including the United States and southern Europe. ${ }^{31}$

The mechanisms of malignant transformation into HCC can be classified into two categories. First, liver cirrhosis progresses because of some underlying reason, in which chromosomal instability via accumulation of somatic alterations and mutations in telomerase reverse transcriptase (one of the "gatekeeper" alterations in the HCC transformation sequence) $)^{32}$ occur, thus leading to a dysplastic nodule that progresses to a malignant tumor and ultimately HCC. ${ }^{33}$ Second, HCC is caused by a specific etiology that directly induces DNA mutagenesis in human cells. ${ }^{34,35}$ Factors related to the first mechanism are alcoholic hepatitis and hepatitis $C$ virus infection, whereas those associated with the second mechanism are hepatitis B virus infection and the presence of aflatoxin. In HCC that develops due to the former mechanism, the alpha-fetoprotein level is low, the degree of differentiation is high, and invasion of the blood vessels is less common. Nonetheless, the presence of underlying cirrhosis is common. In contrast, HCC caused by the latter mechanism is associated with a high alpha-fetoprotein level, a low degree of differentiation, and a strong tendency to invade blood vessels. At the time of diagnosis, deterioration of liver function related to cirrhosis was less severe. The clinical prognosis is generally poorer, and recurrence is more commonly associated with the latter mechanism than with the former mechanism. ${ }^{36-38}$ Zucman-Rossi et al. ${ }^{39}$ classified HCCs that develop according to the first and second mechanisms as the nonproliferation and proliferation classes, respectively.

The vast majority of HCCs that occur in East Asian patients are caused by hepatitis B virus infection, which corresponds to the "proliferation class" according to the classification described above. Proliferation-class HCC is often diagnosed as a locally advanced disease in clinics, and $>80 \%$ of HCCs occurring in China cannot be radically resected at the time of diagnosis. ${ }^{40}$ According to a nationwide random sampling analysis study recently conducted by our team, approximately one-third of patients with HCC in South Korea were diagnosed with stage $\mathrm{C}$ (advanced stage) disease according to the Barcelona Clinic of Liver Cancer staging system, and approximately $70 \%$ of these patients had portal vein invasion. ${ }^{41}$

Major vessel invasion, including portal vein invasion, renders many local treatments difficult to apply. However, EBRT has the ability to target major vessels at a sufficient dose that yields a significant tumor response. Based on SBRT studies on central lung tumors, the major vessel can tolerate $>100$ Gy of irradiation delivered in 2 Gy per fraction. ${ }^{42}$ EBRT is not limited by the challenge of reaching anatomically hardto-reach locations such as major vessels that are located deep inside the body and/or covered by other organs as the radiation directly penetrates such organs. Furthermore, proliferative-class HCCs are typically associated with relatively preserved hepatic function, although the locally advanced tumor is an additional factor supporting the safe application of EBRT. Therefore, many pioneering studies on palliating unresectable HCCs have been conducted in East Asian countries. In our previous meta-analysis ${ }^{29}$ of studies involving 2,513 patients with HCC exhibiting portal vein invasion who underwent radiotherapy, all 26 studies that investigated EBRT were conducted in East Asian countries (Japan, China, Taiwan, and South Korea). The pooled response rates in patients who underwent 3DCRT and SBRT were $70.7 \%$ and $51.3 \%$, respectively. The 1 -year survival rates were $43.8 \%$ and $48.5 \%$, respectively. The rate of grade $\geq 3$ complications was mostly $<10 \%$ or the complication was limited to transient elevation of blood markers after 3DCRT or SBRT. Inferior vena cava involvement was also successfully palliated by EBRT, with a pooled response and local control rates of $59.2 \%$ and $83.8 \%$, respectively. In another meta-analysis by our team; incidences of serious toxicities, including esophagitis or pulmonary embolism, were very rare (1.2\%). ${ }^{43}$

In contrast, non-proliferation-class HCC tends to be accompanied by liver cirrhosis and low- or high-grade dyspla- 
sia and becomes malignant in stages. ${ }^{39}$ When diagnosed, liver cirrhosis is commonly present and liver function often deteriorates. However, non-proliferation-class HCC tends to show less local progression than proliferation-class HCC. Patients with HCC and deteriorated liver function, but not locally advanced lesions, may be suitable candidates for liver transplantation. Of the patients with HCC waiting for transplantation, $24-44 \%$ of patients were dropped from waiting lists owing to disease progression within 1-2 years. ${ }^{44}$ In a recent study, SBRT was used as a bridging therapy to lower the dropout rate and allow $63-100 \%$ of patients to undergo liver transplantation. ${ }^{45}$ Mohamed et al. ${ }^{46}$ compared SBRT, TACE, RFA, and yttrium-90 microspheres as bridging therapy for HCC and reported favorable disease-free survival using all modalities. However, SBRT and yttrium-90 had the lowest rates of serious toxicity. Most studies on SBRT as a bridging therapy have been performed in Western countries. ${ }^{45}$ Table 1 summarizes the clinical and etiological comparisons between non-proliferation- and proliferation-class HCCs and examples of EBRT indications for these diseases.

\section{TREATMENT STRATEGIES ACCORDING TO VARIOUS CLINICAL GUIDELINES}

Currently, more than 30 clinical treatment guidelines for HCC have been published worldwide. The existence of such a large number of guidelines is likely because the disease characteristics of HCC differ by region. The treatment strate- gies must be set in accordance to the situation prevalent in every region. ${ }^{12,47}$ The most commonly used clinical guidelines worldwide are the European Association for the Study of the Liver (EASL), American Association for the Study of Liver Diseases (AASLD), and National Cancer Comprehensive Network (NCCN) guidelines. The EASL and AASLD guidelines were created by the hepatologists' societies in Europe and the United States, respectively, while the NCCN guidelines were created by clinical oncologists (including internal, radiation, and surgical oncologists) from 28 major cancer centers in the United States.

The EASL and AASLD have published various clinical guidelines for HCC and various inflammatory or benign diseases of the liver. Corresponding to the nature of the hepatologists who mainly perform medical interventions, the EASL and AASLD views of HCC treatment are principled and evidence-based. ${ }^{6,48}$ However, the NCCN guidelines give fair consideration to the uniqueness of the field of oncology. Their authors argue that well-designed randomized studies might not always provide a basis for clinical decision making for cancer treatments and that such decisions often need to be made based on observational studies or even clinical experience. $^{49}$

From a conventional perspective, the level of evidence for EBRT for HCC is not high. In clinical practice, radiation oncology departments are not the first to encounter patients for treatment; patients are diagnosed and evaluated by other departments (including gastroenterology or general surgery)

Table 1. Comparison of the non-proliferation and proliferation classes of HCCS

\begin{tabular}{|c|c|c|}
\hline & Non-proliferation class & Proliferation class \\
\hline Common etiology & HCV infection, alcoholic hepatitis & HBV infection \\
\hline Mechanism of malignant formation & $\begin{array}{l}\text { Accumulation of somatic alteration and TERT } \\
\text { mutation }\end{array}$ & Insertion mutagenesis from viral oncoprotein \\
\hline Vascular invasion & Relatively uncommon & Common \\
\hline Clinical features & $\begin{array}{l}\text { Low AFP level, well to moderate differentiation, } \\
\text { common underlying cirrhosis }\end{array}$ & $\begin{array}{l}\text { High AFP level, poor differentiation, relatively } \\
\text { spared liver function }\end{array}$ \\
\hline Common regional distribution & Western countries and Japan & East and Southeast Asia \\
\hline Example of EBRT indication & Bridging liver transplant & Palliation of major vessel involvement \\
\hline
\end{tabular}

Adopted and modified from Gastroenterology 2015;149:1226-1239.e4. ${ }^{39}$

HCC, hepatocellular carcinoma; HCV, hepatitis C virus; HBV, hepatitis B virus; TERT, telomerase reverse transcriptase; AFP, alpha-fetoprotein; EBRT, external beam radiotherapy. 
and are then selectively referred when EBRT is considered necessary. Therefore, the design of randomized or prospective studies is difficult. In addition, the vendor-researcher relationship has not yet been established as strongly as it has in other fields of medicine, because the application of EBRT might not directly provide sustained benefits to vendors. Therefore, there are relatively few prospective or large-scale research studies led by vendors in the field of radiation oncology. ${ }^{50}$

In earlier versions of the EASL and AASLD guidelines published before 2010, EBRT for HCC has been neglected. The 2012 EASL guidelines briefly state that EBRT cannot be used in the treatment of HCC owing to the lack of scientific evidence and high risk of RILD. It can only be used for the palliation of bone metastasis. ${ }^{18}$ The 2010 AASLD guidelines mentioned very little on EBRT ${ }^{51}$ Radiation oncologists were not included as main panelists during the production of these two guidelines. However, because many studies on EBRT have been published since the 2000s, recent EASL guidelines mention its palliative role in patients with portal vein thrombosis (combined with TACE) and the effectiveness of SBRT, although the level of evidence and strength of recommendation is low and weak (C2 per the GRADE system), respectively. ${ }^{6,52}$ The AASLD guidelines also state that EBRT can be considered for unresectable HCC as a potential local modality. However, the level of evidence and strength of recommendation were low and strong ( $\mathrm{C} 1$ per the GRADE system), respectively. ${ }^{52}$ The AASLD guidelines also state that locoregional treatment might be considered for patients with macrovascular invasion or metastatic disease (including EBRT), although they have not specifically recommended a certain modality. ${ }^{48}$ In contrast, the NCCN guidelines suggested EBRT as a therapeutic approach for unresectable HCC along with TACE and RFA since the beginning of 2010. The guidelines issued in the latter half of 2010 actively describe the potent efficacy of SBRT and proton therapy. In the NCCN guidelines issued after 2018, EBRT was suggested as a treatment option for unresectable HCC with the same recommendation level as TACE and RFA (2A: low level of evidence and uniform consensus for application [NCCN categories]). ${ }^{49,53}$ The panelists responsible for these recent NCCN guidelines included surgeons, medical oncologists, radiation oncologists, and hepatologists.

As explained in the preceding section, hepatitis B virus infection is the main cause of HCC in Asia; the disease is commonly diagnosed as a locally advanced tumor, and liver function might relatively less deteriorate. EBRT, which has potent efficacy as a local treatment but may impair liver function, might be suitable for treating such HCCs. Chinese guidelines refer to almost all EBRT indications known to date, including major vessel invasion, preoperative downstaging, bridging for liver transplantation, and extrahepatic metastases. ${ }^{25}$ The evidence grade is 3 per the Oxford system (level of evidence-based on case-control studies), in the Chinese guidelines. ${ }^{12}$ In addition, these guidelines include specialized content related to EBRT, such as methods for tumor targeting and for controlling the tumor's movement via respiration as well as a practical example of dose fractionation. The guidelines of the Korean Liver Cancer Association ${ }^{54}$ also refer to various indications such as post-incomplete TACE (level of evidence and recommendation: B2), portal vein invasion (B2), tumors not amenable to surgery or ablation (C1), and extrahepatic metastases (C1). According to the GRADE classification, B and C represent moderate and low levels of evidence, respectively, while 1 and 2 represent strong and weak recommendations, respectively. ${ }^{12,52}$ The Korean guidelines mention that many practical factors such as liver function indicators or radiation dose restrictions should be considered when applying EBRT. However, the recent Japan Society of Hepatology guidelines for HCC do not include any content regarding EBRT for hepatic tumors, although they briefly state that brain or bone metastases could be indications for palliation. ${ }^{55}$ Hepatitis $\mathrm{C}$ virus infection is the leading cause of HCC in Japan, and tumors are commonly accompanied by liver decompensation at diagnosis. However, the local invasion of the tumor is relatively less severe. Therefore, the efficacy of EBRT might be weaker. We summarized perspectives on EBRT from clinical guidelines that are reputable or regionally representative, including the guidelines explained above but not limited to, in Fig. 3 . 
Estimated age-standardized incidence rates (World) in 2018, liver, males, all ages

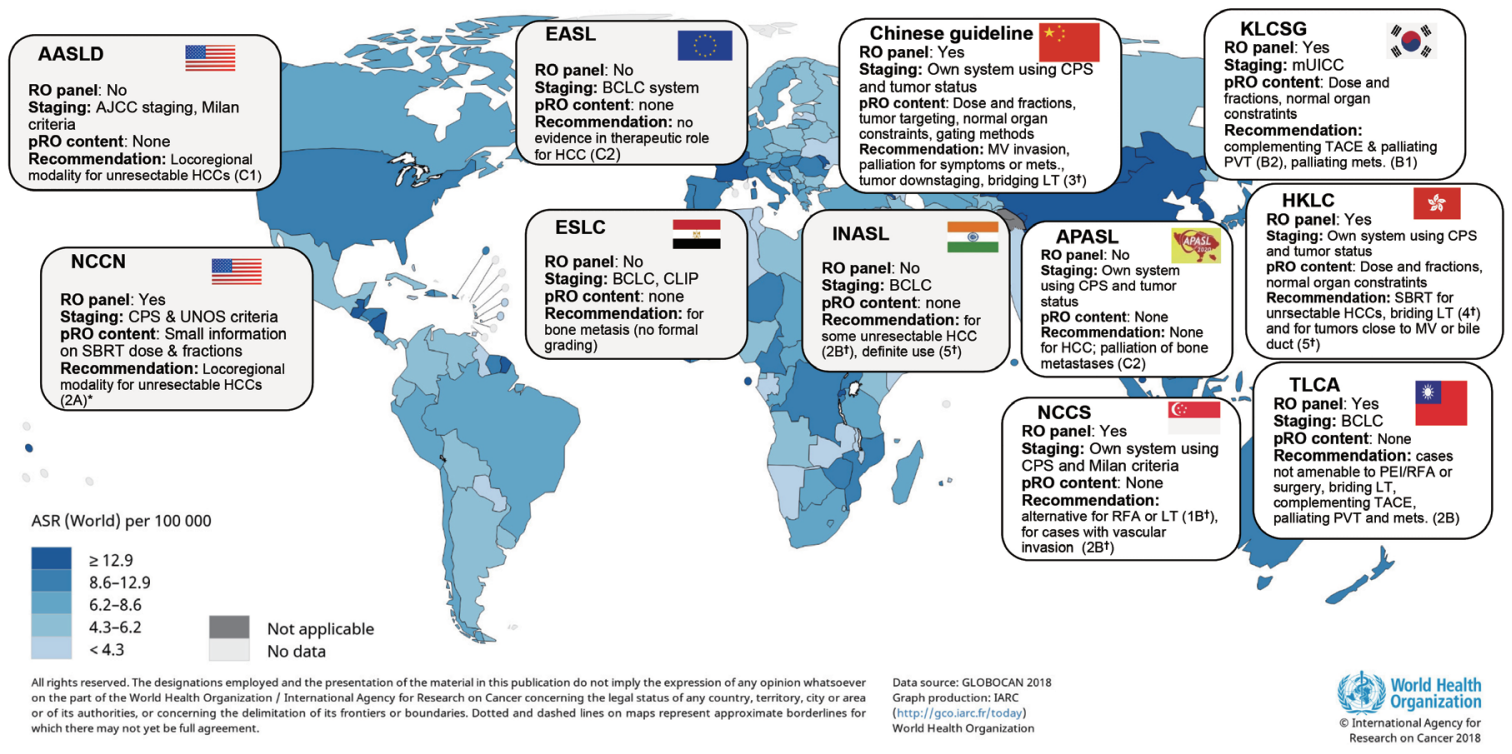

Figure 3. Key summary of selected clinical guidelines for hepatocellular carcinoma (HCC). AASLD, American Association for the Study of Liver Disease; RO, radiation oncology; AJCC, American Joint Committee on Cancer; HCC, hepatocellular carcinoma; EASL, European Association for the Study of the Liver; BCLC, Barcelona Clinic of Liver Cancer; PRO, practical radiation oncology; CPS, Child-Pugh Score; MV, major vessel; LT, liver transplantation; KLCSG, Korea Liver Cancer Study Group; mUICC, modified Union for International Cancer Control; TACE, transarterial chemoembolization; PVT, portal vein thrombosis; NCCN, national cancer comprehensive network; UNOS, United Network for Organ Sharing; SBRT, stereotactic body radiotherapy; ESLC, Egyptian Study of Liver Cancer; CLIP, Cancer of Liver Italian Program; INASL, Indian National Association for the Study of the Liver; APASL, Asia-Pacific Association for the Study of the Liver; HKLC, Hong Kong Liver Cancer study group; NCCS, National Cancer Center Singapore; RFA, radiofrequency ablation; TLCA, Taiwan Liver Cancer Association; PEl, percutaneous alcohol injection; ASR, age-standardized incidence rate. Recommendation levels are according to ${ }^{*} \mathrm{NCCN}$ system, ${ }^{\dagger}$ Oxford system, otherwise GRADE system.

\section{SUMMARY AND PERSPECTIVES}

As a radiation oncologist in East Asia, it is common to encounter patients with HCC who seek treatment with EBRT. According to a recent South Korean surveillance study of various physicians, ${ }^{56}$ more responders consider administering EBRT rather than sorafenib for patients with portal vein thrombosis or incomplete TACE. However, oncologists who specialize in radiation oncology in Western countries are not familiar with the treatment of HCC. They tend to be more aware of hepatic insufficiency that could result from EBRT or have other critical perspectives owing to the low level of evidence.

EBRT played a small role in HCC 30-40 years ago, although with the development of new treatment technologies, the paradigm has significantly changed and EBRT is now a feasible and efficient modality. HCC has different characteristics depending on its etiology. EBRT is more suitable for hepatitis B virus infection-related HCC, which is commonly found to be locally advanced with relatively well-preserved liver function. HCCs associated with hepatitis $\mathrm{C}$ virus infection or benign hepatitis are less suitable candidates for EBRT, although it is a possible modality for bridging liver transplantation. Furthermore, there are critical perspectives due to the low level of evidence of EBRT for HCC. Radiation oncology is a field in which most patients with cancer are selectively referred to specialists. Investor-led research has been relatively scarce because radiation oncology does not provide sustained benefits to vendors through the continued use of drugs or other medical supplies. Therefore, caution should be exercised when attempting to understand the clinical need for EBRT solely based on the conventional level of evidence.

The application of EBRT for HCC has been increasing worldwide, and major guidelines in Western countries are more open to incorporating indications of EBRT compared with that in the past. In the near future, it will be necessary to 
confirm the clinical need for administering EBRT to patients with HCC and to optimize treatment strategies through international academic exchanges and additional clinical studies.

\section{Conflicts of Interest}

The authors declare no conflicts of interest relevant to this article.

\section{Ethics Statement}

This review article is fully based on the articles which was already published and did not involve additional patient participants. Therefore, IRB approval is not necessary.

\section{Funding Statement}

This study was supported by the Research Award of the Korean Liver Cancer Association (2020).

\section{Data Availability}

Data sharing not applicable to this article as no datasets were generated or analyzed during the study.

\section{ORCID}

Chai Hong Rim https://orcid.org/0000-0001-7431-4588

\section{Author Contribution}

CHR contributed to the conceptualization, writing-original draft, and editing, and approved the final manuscript.

\section{References}

1. Sung H, Ferlay J, Siegel RL, Laversanne M, Soerjomataram I, Jemal A, et al. Global Cancer Statistics 2020: GLOBOCAN estimates of incidence and mortality worldwide for 36 cancers in 185 countries. CA Cancer J Clin 2021;71:209-249.

2. Cheung TT, Kwok PC, Chan S, Cheung CC, Lee AS, Lee V, et al. Hong Kong consensus statements for the management of unresectable hepatocellular carcinoma. Liver Cancer 2018;7:40-54.

3. Cho CM, Tak WY, Kweon YO, Kim SK, Choi YH, Hwang YJ, et al. The comparative results of radiofrequency ablation versus surgical resection for the treatment of hepatocellular carcinoma. Korean J Hepatol 2005;11:59-71.

4. Hung HH, Chiou YY, Hsia CY, Su CW, Chou YH, Chiang JH, et al.
Survival rates are comparable after radiofrequency ablation or surgery in patients with small hepatocellular carcinomas. Clin Gastroenterol Hepatol 2011;9:79-86.

5. Llovet JM, Bruix J. Systematic review of randomized trials for unresectable hepatocellular carcinoma: chemoembolization improves survival. Hepatology 2003;37:429-442.

6. European Association for the Study of the Liver. EASL clinical practice guidelines: management of hepatocellular carcinoma. J Hepatol 2018;69:182-236.

7. Dawson LA, Ten Haken RK, Lawrence TS. Partial irradiation of the liver. Semin Radiat Oncol 2001;11:240-246.

8. Seong J. Recent developments in radiotherapy of hepatocellular carcinoma. Korean J Hepatol 2004;10:241-247.

9. Lee J, Shin IS, Yoon WS, Koom WS, Rim CH. Comparisons between radiofrequency ablation and stereotactic body radiotherapy for liver malignancies: meta-analyses and a systematic review. Radiother Oncol 2020;145:63-70.

10. Rim CH, Kim HJ, Seong J. Clinical feasibility and efficacy of stereotactic body radiotherapy for hepatocellular carcinoma: a systematic review and meta-analysis of observational studies. Radiother Oncol 2019;131:135-144.

11. Rim CH, Cheng J, Huang WY, Kimura T, Lee V, Zeng ZC, et al. An evaluation of hepatocellular carcinoma practice guidelines from a radiation oncology perspective. Radiother Oncol 2020;148:73-81.

12. Park S, Yoon WS, Rim CH. Indications of external radiotherapy for hepatocellular carcinoma from updated clinical guidelines: diverse global viewpoints. World J Gastroenterol 2020;26:393-403.

13. Choudhary S, Mitra S, Sharma MK, Khullar P, Saxena U. Resurrection of whole liver radiotherapy-a case report. J Egypt Natl Canc Inst 2017;29:105-108.

14. Lawrence TS, Robertson JM, Anscher MS, Jirtle RL, Ensminger WD, Fajardo LF. Hepatic toxicity resulting from cancer treatment. Int J Radiat Oncol Biol Phys 1995;31:1237-1248.

15. Ingold JA, Reed GB, Kaplan HS, Bagshaw MA. Radiation hepatitis. Am J Roentgenol Radium Ther Nucl Med 1965;93:200-208.

16. Russell AH, Clyde C, Wasserman TH, Turner SS, Rotman M. Accelerated hyperfractionated hepatic irradiation in the management of patients with liver metastases: results of the RTOG dose escalating protocol. Int J Radiat Oncol Biol Phys 1993;27:117-123.

17. Emami B, Lyman J, Brown A, Coia L, Goitein M, Munzenrider JE, et al. Tolerance of normal tissue to therapeutic irradiation. Int J Radiat Oncol Biol Phys 1991;21:109-122.

18. European Association For The Study Of The Liver; European Organisation For Research And Treatment Of Cancer. EASL-EORTC clinical practice guidelines: management of hepatocellular carcinoma. J Hepatol 2012;56:908-943.

19. Dawson LA, Normolle D, Balter JM, McGinn CJ, Lawrence TS, Ten Haken RK. Analysis of radiation-induced liver disease using the Lyman NTCP model. Int J Radiat Oncol Biol Phys 2002;53:810-821. 
20. Pan CC, Kavanagh BD, Dawson LA, Li XA, Das SK, Miften M, et al. Radiation-associated liver injury. Int J Radiat Oncol Biol Phys 2010;76:S94-S100.

21. Kim TH, Kim DY, Park JW, Kim SH, Choi Jl, Kim HB, et al. Dosevolumetric parameters predicting radiation-induced hepatic toxicity in unresectable hepatocellular carcinoma patients treated with three-dimensional conformal radiotherapy. Int J Radiat Oncol Biol Phys 2007;67:225-231.

22. Schefter $T E$, Kavanagh BD, Timmerman RD, Cardenes HR, Baron A, Gaspar LE. A phase I trial of stereotactic body radiation therapy (SBRT) for liver metastases. Int J Radiat Oncol Biol Phys 2005;62:1371-1378.

23. Koay EJ, Owen D, Das P. Radiation-induced liver disease and modern radiotherapy. Semin Radiat Oncol 2018;28:321-331.

24. Korean Liver Cancer Association (KLCA); National Cancer Center (NCC). 2018 Korean Liver Cancer Association-National Cancer Center Korea practice guidelines for the management of hepatocellular carcinoma. Korean J Radiol 2019;20:1042-1113.

25. Zhou J, Sun HC, Wang Z, Cong WM, Wang JH, Zeng MS, et al. Guidelines for diagnosis and treatment of primary liver cancer in China (2017 Edition). Liver Cancer 2018;7:235-260.

26. Marks LB, Yorke ED, Jackson A, Ten Haken RK, Constine LS, Eisbruch $A$, et al. Use of normal tissue complication probability models in the clinic. Int J Radiat Oncol Biol Phys 2010;76(3 Suppl):S10S19.

27. Park HC, Seong J, Han KH, Chon CY, Moon YM, Suh CO. Doseresponse relationship in local radiotherapy for hepatocellular carcinoma. Int J Radiat Oncol Biol Phys 2002;54:150-155.

28. Rim CH, Yang DS, Park YJ, Yoon WS, Lee JA, Kim CY. Effectiveness of high-dose three-dimensional conformal radiotherapy in hepatocellular carcinoma with portal vein thrombosis. Jpn J Clin Oncol 2012;42:721-729.

29. $\operatorname{Rim} \mathrm{CH}, \mathrm{Kim}$ CY, Yang DS, Yoon WS. Comparison of radiation therapy modalities for hepatocellular carcinoma with portal vein thrombosis: a meta-analysis and systematic review. Radiother Oncol 2018;129:112-122.

30. Maucort-Boulch D, de Martel C, Franceschi S, Plummer M. Fraction and incidence of liver cancer attributable to hepatitis B and $C$ viruses worldwide. Int J Cancer 2018;142:2471-2477.

31. Choo SP, Tan WL, Goh BKP, Tai WM, Zhu AX. Comparison of hepatocellular carcinoma in Eastern versus Western populations. Cancer 2016;122:3430-3446.

32. Schulze K, Imbeaud S, Letouzé E, Alexandrov LB, Calderaro J, Rebouissou $S$, et al. Exome sequencing of hepatocellular carcinomas identifies new mutational signatures and potential therapeutic targets. Nat Genet 2015;47:505-511.

33. International Consensus Group for Hepatocellular Neoplasia. Pathologic diagnosis of early hepatocellular carcinoma: a report of the international consensus group for hepatocellular neoplasia. Hepatology 2009;49:658-664.

34. Neuveut C, Wei Y, Buendia MA. Mechanisms of HBV-related hepatocarcinogenesis. J Hepatol 2010;52:594-604.

35. Brechot C, Pourcel C, Louise A, Rain B, Tiollais P. Presence of integrated hepatitis $B$ virus DNA sequences in cellular DNA of human hepatocellular carcinoma. Nature 1980;286:533-535.

36. Villanueva A, Hoshida Y, Battiston C, Tovar V, Sia D, Alsinet C, et al. Combining clinical, pathology, and gene expression data to predict recurrence of hepatocellular carcinoma. Gastroenterology 2011;140:1501-1512.e2.

37. Lee JS, Chu IS, Heo J, Calvisi DF, Sun Z, Roskams T, et al. Classification and prediction of survival in hepatocellular carcinoma by gene expression profiling. Hepatology 2004;40:667-676.

38. Chiang DY, Villanueva A, Hoshida Y, Peix J, Newell P, Minguez B, et al. Focal gains of VEGFA and molecular classification of hepatocellular carcinoma. Cancer Res 2008;68:6779-6788.

39. Zucman-Rossi J, Villanueva A, Nault JC, Llovet JM. Genetic landscape and biomarkers of hepatocellular carcinoma. Gastroenterology 2015;149:1226-1239.e4.

40. Wu Q, Qin SK. Features and treatment options of Chinese hepatocellular carcinoma. Chin Clin Oncol 2013;2:38.

41. Lee J, Yoon WS, Koom WS, Rim CH. Role of local treatment including radiotherapy in Barcelona Clinic of Liver Cancer stage C patients: a nationwide cohort analysis in South Korea. Cancer Manag Res 2019;11:1373-1382.

42. Rim CH, Yoon WS. Leaflet manual of external beam radiation therapy for hepatocellular carcinoma: a review of the indications, evidences, and clinical trials. Onco Targets Ther 2018;11:28652874.

43. Rim CH, Kim CY, Yang DS, Yoon WS. External beam radiation therapy to hepatocellular carcinoma involving inferior vena cava and/ or right atrium: a meta-analysis and systemic review. Radiother Oncol 2018;129:123-129.

44. Yao FY, Bass NM, Nikolai B, Davern TJ, Kerlan R, Wu V, et al. Liver transplantation for hepatocellular carcinoma: analysis of survival according to the intention-to-treat principle and dropout from the waiting list. Liver Transpl 2002;8:873-883.

45. Murray LJ, Dawson LA. Advances in stereotactic body radiation therapy for hepatocellular carcinoma. Semin Radiat Oncol 2017; 27:247-255.

46. Mohamed M, Katz AW, Tejani MA, Sharma AK, Kashyap R, Noel $M S$, et al. Comparison of outcomes between SBRT, yttrium-90 radioembolization, transarterial chemoembolization, and radiofrequency ablation as bridge to transplant for hepatocellular carcinoma. Adv Radiat Oncol 2015;1:35-42.

47. Yoon SM. External beam radiotherapy for hepatocellular carcinoma: a review of the current guidelines in the East and the West. 
J Liver Cancer 2021;21:25-33.

48. Heimbach JK, Kulik LM, Finn RS, Sirlin CB, Abecassis MM, Roberts $L R$, et al. AASLD guidelines for the treatment of hepatocellular carcinoma. Hepatology 2018;67:358-380.

49. National Comprehensive Cancer Network (NCCN). NCCN levels of evidence and consensus for recommendations [Internet]. Plymouth Meeting (PA): National Comprehensive Cancer Network; [cited 2020 Oct 21]. Available from: https://www.nccn.org/professionals/ development.aspx.

50. Jagsi $R$, Sheets $N$, Jankovic $A$, Motomura AR, Amarnath $S$, Ubel $P A$. Frequency, nature, effects, and correlates of conflicts of interest in published clinical cancer research. Cancer 2009;115:27832791.

51. Bruix J, Sherman M; American Association for the Study of Liver Diseases. Management of hepatocellular carcinoma: an update. Hepatology 2011;53:1020-1022.

52. Schunemann H, Brozek J, Guyatt G, Oxman A. GRADE Handbook: handbook for grading the quality of evidence and the strength of recommendations using the GRADE approach [Internet]. Hamilton
(CA): GRADE working group; [cited 2020 Oct 21]. Available from: https://gdt.gradepro.org/app/handbook/handbook.html.

53. National Comprehensive Cancer Network (NCCN). NCCN guidelines version 1. 2018, hepatobiliary cancers [Internet]. Plymouth Meeting (PA): National Comprehensive Cancer Network; [cited 2020 Oct 21]. Available from: https://www.nccn.org/professionals/ physician_gls/pdf/hepatobiliary.pdf.

54. Korean Liver Cancer Association; National Cancer Center. 2018 Korean Liver Cancer Association-National Cancer Center Korea practice guidelines for the management of hepatocellular carcinoma. Gut Liver 2019;13:227-299.

55. Kokudo N, Takemura N, Hasegawa K, Takayama T, Kubo S, Shimada $\mathrm{M}$, et al. Clinical practice guidelines for hepatocellular carcinoma: the Japan Society of Hepatology 2017 (4th JSH-HCC guidelines) 2019 update. Hepatol Res 2019;49:1109-1113.

56. Lee JS, Kim BK, Kim SU, Park JY, Ahn SH, Seong JS, et al. A survey on transarterial chemoembolization refractoriness and a real-world treatment pattern for hepatocellular carcinoma in Korea. Clin Mol Hepatol 2020;26:24-32. 